\title{
Studies towards the synthesis of hyperireflexolide A
}

\author{
G. Hari Mangeswara Rao ${ }^{1,2}$
}

\author{
Full Research Paper \\ Address: \\ 1Department of Chemistry, Indian Institute of Technology Kanpur, \\ Kanpur-208016, India and 2Department of Chemistry, Texila \\ American University, Georgetown, Guyana \\ Email: \\ G. Hari Mangeswara Rao - harimangesh@gmail.com

\section{Keywords:} \\ alkylation; allylation; cross metathesis; hyperireflexolide A; \\ spiroterpene
}

\author{
Beilstein J. Org. Chem. 2018, 14, 2106-2111. \\ doi:10.3762/bjoc. 14.185
}

Received: 27 April 2018

Accepted: 25 July 2018

Published: 13 August 2018

Associate Editor: B. Stoltz

(c) 2018 Rao; licensee Beilstein-Institut.

License and terms: see end of document.

\begin{abstract}
The first approach to hyperireflexolide A, based on the synthesis of $\gamma$-lactone-fused cyclopentane $\mathbf{5}$, a functionalized key intermediate, is presented. Compound $\mathbf{5}$ is involved in hydrolysis, $\alpha$-allylation at C- 8 and $\alpha$-methylation at $\mathrm{C}-10$ stereoselectively from the convex face. Then it is subjected to cross metathesis to give $\alpha, \beta$-unsaturated ketone $\mathbf{1 1}$ as precursor in the total synthesis of hyperireflexolide A.
\end{abstract}

\section{Introduction}

Hyperireflexolide A (1) [1] is a spiroterpenoid, isolated from hypericum reflexum, plants of the genus hypericum (Figure 1). Hyperireflexolide A is widely used in folk medicine, displays antifungal [2] and cytotoxic activities [3].

$\gamma$-Lactone-fused cyclopentanes are of vital importance in organic synthesis and are the most abundant substructures found in various naturally occurring molecules $[4,5]$. A cis-cyclopentane ring-fused $\gamma$-lactone is the key structural unit of many complex and challenging biologically active natural products [6-19] The $\gamma$-lactone-fused cyclopentane ring system is also an important component for the synthesis of a variety of cyclopentanoid natural and unnatural products [20-24].

In the literature, numerous synthetic methods are reported to attain $\gamma$-lactone-fused cyclopentanes [25-31]. Earlier from our lab, we reported a short and efficient methodology for the synthesis of $\gamma$-lactone-fused cyclopentane 5 [32]. The cis-ring junction of this carbocylic ring system offers a high degree of selectivity for the assemblage of various substituents on the convex surface. The lactone part can serve as a useful tool to append various side chains.

\section{Results and Discussion}

The presence of a $\gamma$-lactone-fused cyclopentane moiety in hyperireflexolide A (1) attracted our attention. In fact, the ketal moiety in $\mathbf{5}$ could not only act as a surrogate for C-9 carbonyl but also facilitate installation of an angular methyl group.

The retrosynthetic analysis for hyperireflexolide A is depicted in Scheme 1. We envisioned that hyperireflexolide A (1) could be synthesized by metal-catalyzed opening of the epoxide 2 


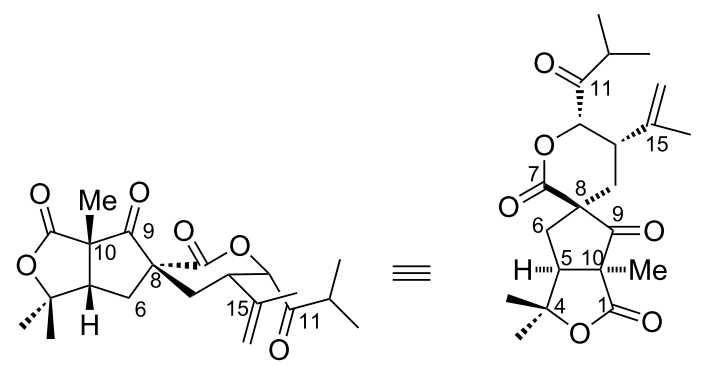

hyperireflexolide $\mathrm{A}$

(1)

Figure 1: Hyperireflexolide A.

with 2-bromopropene followed by lactonization. Enone $\mathbf{3}$ could be synthesized from 4 by installation of the methyl group at C-10 followed by cross metathesis reaction. Compound 4 could be obtained from the $\gamma$-lactone-fused cyclopentane 5 by deprotection of C-9 followed by allylation at C-8.

Previously, we reported from our laboratory the synthesis of $\gamma$-lactone-fused cyclopentane derivative 5 from the respective Diels-Alder adduct in 5 steps with an overall yield of 29\% [32]. Hydrolysis of dimethyl ketal 5 with $\mathrm{MeSO}_{3} \mathrm{H}$ in 1,2-DCE furnished $\gamma$-lactone-fused cyclopentanone 6 in 97\% yield. Cyclopentanone 6 exists in its tautomeric enol form 7, observed in the ${ }^{1} \mathrm{H}$ NMR spectrum after column chromatographic purification (6 and 7 were not separated) as represented in Scheme 2 [33].

In order to check the feasibility of the alkylation reaction of $\gamma$-lactone-fused $\beta$-ketoester 6 , initially a mixture of $\mathbf{6}$ and 7 was subjected to methylation using 1.1 equiv of $\mathrm{K}_{2} \mathrm{CO}_{3}$ in the presence of methyl iodide (MeI). The $\alpha$-methylated $\beta$-ketoester $\mathbf{8}$ was obtained in good yield. In the ${ }^{1} \mathrm{H}$ NMR, 8 showed a signal at $3.70 \mathrm{ppm}$ as doublet for $\mathrm{C}-10$ (ring junction) proton confirming the selective methylation at C-8. Further, installation of the methyl group at $\mathrm{C}-10$ was achieved by treatment of 8 with 1.1 equiv of $\mathrm{K}_{2} \mathrm{CO}_{3}$ in the presence of $\mathrm{MeI}$ to give bismethylated $\gamma$-lactone-fused $\beta$-ketoester 9 in $72 \%$ yield

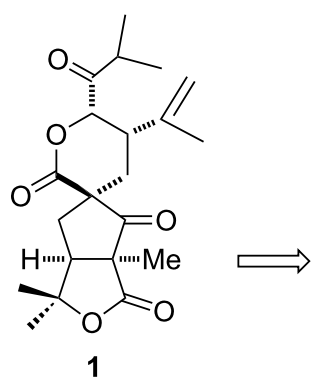

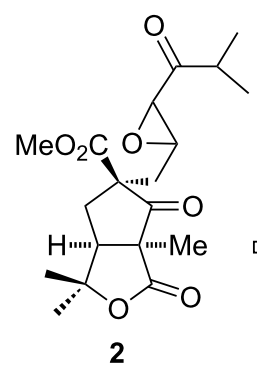<smiles>C=CC=CC(=O)C(C)C</smiles>

hyperireflexolide $\mathrm{A}$

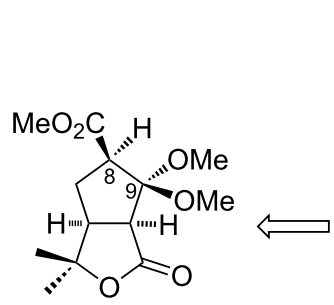

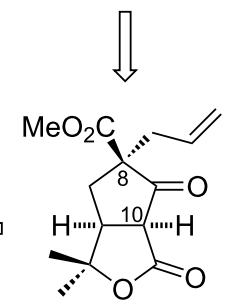

4

Scheme 1: Retrosynthetic strategy.

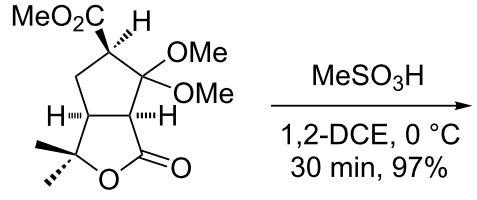

5<smiles>COC(=O)[C@H]1C[C@H]2C(=O)O[C@@H](C)[C@H]2C(=O)O1</smiles>

6<smiles>CC(=O)C1=C(O)[C@H]2C(=O)OC(C)(C)[C@H]2C1</smiles>

7 
(Scheme 3). These results demonstrated that regioselective alkylation at the two sites were possible. Notably, one diastereomeric product was isolated from these bis-alkylation reactions due to favorable attack from the less hindered convex face of 6 [10] to give $\alpha, \alpha^{\prime}$-cis stereochemistry.

Now the stage has been set for allylation of $\gamma$-lactone fused cyclopentanone $\mathbf{6}$. Treatment of 6 with $\mathrm{K}_{2} \mathrm{CO}_{3}$ in the presence of allyl bromide at $0{ }^{\circ} \mathrm{C}$ afforded $\alpha$-allylated $\gamma$-lactone-fused $\beta$-ketoester 4 in $88 \%$ yield. In the ${ }^{1} \mathrm{H}$ NMR a signal appeared at $3.64 \mathrm{ppm}$ as doublet for ring junction (C-10) proton confirmed that selective allylation occurred at $\mathrm{C}-8$. Compound 4 was then subjected to methylation at $\mathrm{C}-10$ using $\mathrm{K}_{2} \mathrm{CO}_{3}$ and MeI to obtain the requisite $\gamma$-lactone fused cyclopentanone $\mathbf{1 0}$ in excellent yield (Scheme 4). Allylation and methylation both were occurred stereoselectively from the convex face to give $\alpha, \alpha^{\prime}$-cis stereochemistry. The allyl derivative $\mathbf{1 0}$ was then subjected to cross metathesis reaction with ethyl vinyl ketone. Initially, the reaction performed using Grubbs' 1 st generation catalyst
(3-20 mol \%) was unsuccessful. Treatment of $\mathbf{1 0}$ with ethyl vinyl ketone using Grubbs' 2nd generation catalyst (3 $\mathrm{mol} \%$ ) in the presence of $\mathrm{CH}_{2} \mathrm{Cl}_{2}$ furnished $(E)$-enone $\mathbf{1 1}$ in $77 \%$ yield as shown in Scheme 4. In the ${ }^{1} \mathrm{H}$ NMR spectrum, 11 showed a signal at $6.13 \mathrm{ppm}$ as doublet with coupling constant $15.8 \mathrm{~Hz}$ (trans-configuration) for the $\alpha$-proton of enone [34-37].

After successfully synthesizing the side chain via cross-metathesis, our next task was the steresoselective epoxidation of (E)-enone 11. Unfortunately, the stereoselective epoxidation of 11 under basic conditions were unsuccessful [38], which prevented completion of the proposed synthetic sequence.

\section{Conclusion}

In conclusion, synthetic studies towards hyperireflexolide A, the synthetic precursor $\alpha, \beta$-unsaturated ketone 11 was synthesized. Failure of the stereoselective epoxidation of $\mathbf{1 1}$ prevented completion of the proposed synthetic sequence. Future studies

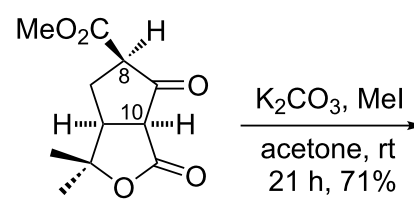

6

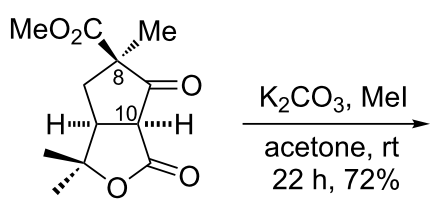

8

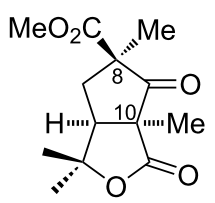

9

Scheme 3: Alkylation of $y$-lactone-fused $\beta$-ketoester 6.

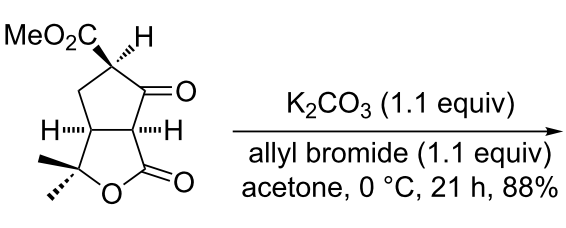

6

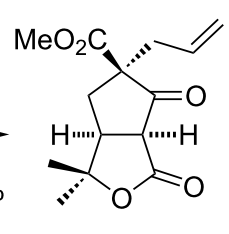

4

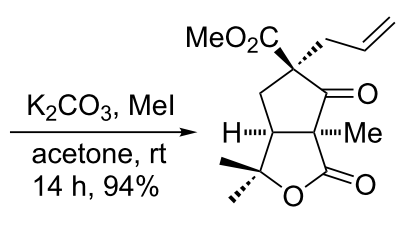

10

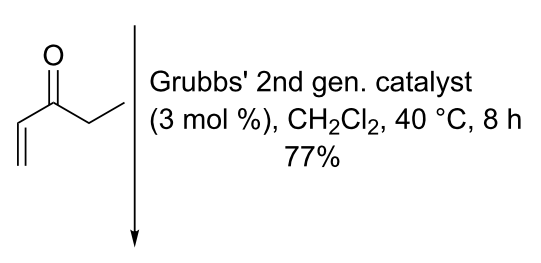<smiles>CCC(=O)/C=C/[C@@]1(C(C)=O)C[C@H]2[C@@H](C)OC(=O)[C@]2(C)C1=O</smiles>

11

Scheme 4: Synthesis of $\alpha, \beta$-unsaturated ketone 11. 
will include the stereoselective epoxidation of $\mathbf{1 1}$ followed by opening of the epoxide and lactonization or 1,4-nucleophilic addition to the $\alpha, \beta$-unsaturated ketone $\mathbf{1 1}$ followed by epoxidation of the resulted enolate with subsequent lactonization to achieve hyperireflexolide A (1).

\section{Experimental}

\section{General methods}

All the reactions were performed in oven dried apparatus and the reaction mixtures were magnetically stirred. Thin-layer chromatography was performed on Acme and Spectrochem Silica gel (Mumbai, India) coated on microscopic slides. Visualization of spots was effected by exposure to iodine or spraying with $4 \%$ ethanolic $\mathrm{H}_{2} \mathrm{SO}_{4}$ and charring. Column chromatography was performed using Acme's silica gel (100-200 mesh), and ethyl acetate/hexane was used as eluent. Evaporation of solvents was performed at reduced pressure using a Büchi rotary evaporator.

Melting points were recorded on JSGW melting point apparatus and are uncorrected. Infrared spectra were recorded on Perkin-Elmer 1320 and Shimadzu 420 spectrophotometers as $\mathrm{KBr}$ pellets (solids), or as thin films on $\mathrm{NaCl}$ flats (liquids). ${ }^{1} \mathrm{H}$ NMR spectra were recorded at $400 \mathrm{MHz}$ on a JEOL spectrometer unless otherwise mentioned $(500 \mathrm{MHz})$. Data are reported as follows: $(\mathrm{br}=$ broad, $\mathrm{s}=$ singlet, $\mathrm{d}=$ doublet, $\mathrm{t}=$ triplet, $\mathrm{q}=$ quartet, $\mathrm{m}=$ multiplet; integration; coupling con$\operatorname{stant}(\mathrm{s})$ in $\mathrm{Hz}$; assignment). Chemical shifts are reported in ppm, and coupling constants are reported in Hz. Proton decoupled ${ }^{13} \mathrm{C}$ NMR spectra were recorded at $100 \mathrm{MHz}$ $(125 \mathrm{MHz})$ on a JEOL spectrometer. Samples for NMR were made in $\mathrm{CDCl}_{3}$. Tetramethylsilane was used as the internal standard.

Commercial grade solvents were distilled before use. Ethyl acetate was distilled over anhydrous sodium carbonate. Dichloromethane and dichloroethane (1,2-DCE) were distilled over phosphorous pentoxide and stored over $4 \AA$ molecular sieves. Acetone was distilled over anhydrous $\mathrm{K}_{2} \mathrm{CO}_{3}$. Methanol was refluxed and distilled over magnesium turnings and stored over $4 \AA$ molecular sieves. Distilled water was used for aqueous reactions and aqueous work-up.

\section{Experimental procedures and analytical data} Methyl 1,1,5-trimethyl-3,4-dioxohexahydro-1 $\mathrm{H}$-cyclopenta[c]furan-5-carboxylate (8): To a solution of the $\gamma$-lactonefused cyclopentanone $\mathbf{6}(16 \mathrm{mg}, 0.07 \mathrm{mmol})$ in dry acetone $(0.4 \mathrm{~mL})$ under argon was added anhydrous $\mathrm{K}_{2} \mathrm{CO}_{3}(9.7 \mathrm{mg}$, $0.07 \mathrm{mmol})$ and methyl iodide $(12 \mathrm{mg}, 0.08 \mathrm{mmol})$ at $0{ }^{\circ} \mathrm{C}$. The reaction was stirred at room temperature for $21 \mathrm{~h}$. Completion of the reaction was monitored by tlc. The reaction was diluted with water $(3 \mathrm{~mL})$ and the organic layer was extracted with EtOAc $(3 \times 3 \mathrm{~mL})$. The combined organic layers were washed with brine solution $(2 \mathrm{~mL})$ and dried over anhydrous $\mathrm{Na}_{2} \mathrm{SO}_{4}$. The solvent was concentrated in vacuo to furnish a residue which was purified by silica gel column chromatography (25\% EtOAc/hexane) to afford $\mathbf{8}$ in $71 \%$ yield. Viscous liquid; ${ }^{1} \mathrm{H}$ NMR (400 MHz, $\left.\mathrm{CDCl}_{3}\right) \delta 3.72\left(\mathrm{~s}, 3 \mathrm{H}, \mathrm{CO}_{2} \mathrm{Me}\right), 3.70(\mathrm{~d}$, $J=1.9 \mathrm{~Hz}, 1 \mathrm{H}), 2.98-2.91(\mathrm{~m}, 1 \mathrm{H}), 2.52(\mathrm{dd}, J=13.2,11.2 \mathrm{~Hz}$, 1H), 2.11-2.04 (m, 1H), 1.50 (s, 3H, Me), 1.47 (s, 3H, Me), 1.37 (s, 3H, Me); ${ }^{13} \mathrm{C}$ NMR (100 MHz, $\left.\mathrm{CDCl}_{3}\right) \delta 203.4(\mathrm{C}=\mathrm{O})$, 171.0, 167.7, 84.5, 58.0, 55.6, 52.8, 44.9, 35.1, 29.3, 22.3, 18.7; IR (neat): 2900, 1760,1740,1440 $\mathrm{cm}^{-1}$; HRMS $(\mathrm{m} / \mathrm{z}):[\mathrm{M}]^{+}$ calcd for $\mathrm{C}_{12} \mathrm{H}_{16} \mathrm{O}_{5}, 240.0998$; found, 240.1001 .

Methyl 1,1,3a,5-tetramethyl-3,4-dioxohexahydro- $1 H$-cyclopenta[c]furan-5-carboxylate (9): To a solution of the $\gamma$-lactone-fused cyclopentanone $8(15 \mathrm{mg}, 0.07 \mathrm{mmol})$ in dry acetone $(0.4 \mathrm{~mL})$ under argon was added anhydrous $\mathrm{K}_{2} \mathrm{CO}_{3}$ $(9.7 \mathrm{mg}, 0.07 \mathrm{mmol})$ and methyl iodide $(12 \mathrm{mg}, 0.08 \mathrm{mmol})$ at $0{ }^{\circ} \mathrm{C}$. The reaction was stirred at room temperature for $22 \mathrm{~h}$. Completion of the reaction was monitored by tlc. The reaction was diluted with water $(3 \mathrm{~mL})$ and the organic layer was extracted with EtOAc $(3 \times 3 \mathrm{~mL})$. The combined organic layers were washed with brine solution $(2 \mathrm{~mL})$ and dried over anhydrous $\mathrm{Na}_{2} \mathrm{SO}_{4}$. The solvent was concentrated in vacuo to furnish a residue which was purified by silica gel column chromatography (20\% EtOAc/hexane) to afford 9 in $72 \%$ yield. ${ }^{1} \mathrm{H}$ NMR (400 MHz, $\left.\mathrm{CDCl}_{3}\right) \delta 3.70\left(\mathrm{~s}, 3 \mathrm{H}, \mathrm{CO}_{2} \mathrm{Me}\right), 2.66(\mathrm{t}$, $J=8.6 \mathrm{~Hz}, 1 \mathrm{H}), 2.55(\mathrm{dd}, J=13.9,8.8 \mathrm{~Hz}, 1 \mathrm{H}), 2.05(\mathrm{dd}$, $J=13.9,8.4 \mathrm{~Hz}, 1 \mathrm{H}), 1.64$ (s, 3H, Me), 1.60 (s, 3H, Me), 1.49 (s, 3H, Me), 1.36 (s, 3H, Me); ${ }^{13} \mathrm{C}$ NMR (100 MHz, $\left.\mathrm{CDCl}_{3}\right)$ $\delta 201.4(\mathrm{C}=\mathrm{O}), 169.3,168.4,80.2,59.2,56.4,53.5,44.4,36.5$, 28.7, 22.6, 19.8, 18.7; IR (neat): 2900, 1760, 1740, $1440 \mathrm{~cm}^{-1}$; $\operatorname{HRMS}(\mathrm{m} / \mathrm{z}):[\mathrm{M}]^{+}$calcd for $\mathrm{C}_{13} \mathrm{H}_{18} \mathrm{O}_{5}, 254.1154$; found, 254.1155 .

Methyl 5-allyl-1,1-dimethyl-3,4-dioxohexahydro- $1 H$-cyclopenta[c]furan-5-carboxylate (4): To a solution of the $\gamma$-lactone-fused cyclopentanone $6(75 \mathrm{mg}, 0.331 \mathrm{mmol})$ in dry acetone $(1 \mathrm{~mL})$ under argon was added anhydrous $\mathrm{K}_{2} \mathrm{CO}_{3}$ (55 mg, $0.3972 \mathrm{mmol}$ ) and allyl bromide (48 mg, $0.3972 \mathrm{mmol}$ ) at $0{ }^{\circ} \mathrm{C}$. The reaction was stirred at $0{ }^{\circ} \mathrm{C}$ for $21 \mathrm{~h}$. Completion of the reaction was monitored by tlc. The reaction was diluted with water $(4 \mathrm{~mL})$ and the organic layer was extracted with EtOAc $(3 \times 5 \mathrm{~mL})$. The combined organic layers were washed with brine solution $(3 \mathrm{~mL})$ and dried over anhydrous $\mathrm{Na}_{2} \mathrm{SO}_{4}$. The solvent was concentrated in vacuo to furnish a residue which was purified by silica gel column chromatography (15\% EtOAc/hexane) to afford 4 in $88 \%$ yield. Viscous liquid; ${ }^{1} \mathrm{H}$ NMR (500 MHz, $\left.\mathrm{CDCl}_{3}\right) \delta$ 5.72-5.64 (m, 1H), 5.18-5.12 $(\mathrm{m}, 2 \mathrm{H}), 3.71\left(\mathrm{~s}, 3 \mathrm{H}, \mathrm{CO}_{2} \mathrm{Me}\right), 3.64(\mathrm{~d}, J=7.2 \mathrm{~Hz}, 1 \mathrm{H})$, 
2.86-2.80 (m, 1H), $2.70(\mathrm{dd}, J=13.8,6.5 \mathrm{~Hz}, 1 \mathrm{H}), 2.50-2.45$ $(\mathrm{m}, 1 \mathrm{H}), 2.35-2.30(\mathrm{~m}, 1 \mathrm{H}), 2.25-2.20(\mathrm{~m}, 1 \mathrm{H}), 1.50(\mathrm{~s}, 3 \mathrm{H}$, $\mathrm{Me}), 1.44(\mathrm{~s}, 3 \mathrm{H}, \mathrm{Me}) ;{ }^{13} \mathrm{C} \mathrm{NMR}\left(125 \mathrm{MHz}, \mathrm{CDCl}_{3}\right) \delta 202.6$, 169.9, 167.8, 131.8, 120.2, 84.8, 61.8, 56.2, 53.0, 44.5, 37.3, 31.2, 29.4, 22.3; IR (neat): 2900, 1760, 1720, $1420 \mathrm{~cm}^{-1}$; $\operatorname{HRMS}(\mathrm{m} / \mathrm{z}):[\mathrm{M}]^{+}$calcd for $\mathrm{C}_{14} \mathrm{H}_{18} \mathrm{O}_{5}, 266.1154$; found, 266.1150 .

Methyl 5-allyl-1,1,3a-trimethyl-3,4-dioxohexahydro-1 $H$ cyclopenta $[c]$ furan-5-carboxylate (10): To a solution of the $\gamma$-lactone-fused cyclopentanone 4 (29 mg, $0.109 \mathrm{mmol})$ in dry acetone $(0.6 \mathrm{~mL})$ under argon was added anhydrous $\mathrm{K}_{2} \mathrm{CO}_{3}$ (16.5 mg, $0.1199 \mathrm{mmol})$ and methyl iodide $(18.5 \mathrm{mg}$, $0.1308 \mathrm{mmol}$ ) at $0{ }^{\circ} \mathrm{C}$. The reaction was stirred at room temperature for $14 \mathrm{~h}$. Completion of the starting material was monitored by tlc. The reaction was diluted with water $(3 \mathrm{~mL})$ and the organic layer was extracted with EtOAc $(3 \times 4 \mathrm{~mL})$. The combined organic layers were washed with brine solution $(3 \mathrm{~mL})$ and dried over anhydrous $\mathrm{Na}_{2} \mathrm{SO}_{4}$. The solvent was concentrated in vacuo to furnish a residue which was purified by silica gel column chromatography $(10 \%$ EtOAc/hexane $)$ to afford 10 in $94 \%$ yield. Viscous liquid; ${ }^{1} \mathrm{H}$ NMR $(500 \mathrm{MHz}$, $\left.\mathrm{CDCl}_{3}\right) \delta 5.69-5.61(\mathrm{~m}, 1 \mathrm{H}), 5.17-5.11(\mathrm{~m}, 2 \mathrm{H}), 3.69(\mathrm{~s}, 3 \mathrm{H}$, OMe), 2.66 (dd, $J=13.9,6.5 \mathrm{~Hz}, 1 \mathrm{H}), 2.57(\mathrm{t}, J=8.5 \mathrm{~Hz}, 1 \mathrm{H})$, $2.49(\mathrm{dd}, J=14.0,8.5 \mathrm{~Hz}, 1 \mathrm{H}), 2.35$ (dd, $J=13.9,7.5 \mathrm{~Hz}, 1 \mathrm{H})$, 2.19 (dd, $J=14.0,8.5 \mathrm{~Hz}, 1 \mathrm{H}), 1.59$ (s, 3H, Me), 1.53 (s, 3H, $\mathrm{Me}), 1.50$ (s, 3H, Me); ${ }^{13} \mathrm{C}$ NMR $\left(100 \mathrm{MHz}, \mathrm{CDCl}_{3}\right) \delta 206.0$, 171.8, 170.3, 131.9, 120.1, 84.0, 60.7, 60.1, 52.8, 50.4, 37.8, 30.5, 30.2, 23.7, 22.5; IR (neat): 2900, 1760, 1740, $1460 \mathrm{~cm}^{-1}$; HRMS $(\mathrm{m} / z):[\mathrm{M}]^{+}$calcd for $\mathrm{C}_{15} \mathrm{H}_{20} \mathrm{O}_{5}, 280.1311$; found, 280.1310 .

Methyl 1,1,3a-trimethyl-3,4-dioxo-5-((E)-4-oxohex-2enyl)hexahydro- $1 H$-cyclopenta[c] furan-5-carboxylate (11): To a solution of the compound $\mathbf{1 0}(110 \mathrm{mg}, 0.327 \mathrm{mmol})$ in $\mathrm{CH}_{2} \mathrm{Cl}_{2}(0.8 \mathrm{~mL})$ was added ethyl vinyl ketone $(31 \mathrm{mg}$, $0.3597 \mathrm{mmol}$ ) and $3 \mathrm{~mol} \%$ Grubbs' 2nd generation catalyst $(8.5 \mathrm{mg}, 0.00981 \mathrm{mmol})$, then heated the reaction mixture at $40{ }^{\circ} \mathrm{C}$ for $8 \mathrm{~h}$ (monitored by tlc). The solvent was concentrated in vacuo to furnish a residue which was purified by silica gel column chromatography (15\% EtOAc/hexane) to afford $\mathbf{1 1}$ in $77 \%$ yield. Viscous liquid; ${ }^{1} \mathrm{H}$ NMR $\left(500 \mathrm{MHz}, \mathrm{CDCl}_{3}\right)$ $\delta$ 6.67-6.61 (m, 1H), 6.13 (d, $J=15.8 \mathrm{~Hz}, 1 \mathrm{H},-\mathrm{C}(\mathrm{O})-\mathrm{CH}=\mathrm{C}-)$, $3.70\left(\mathrm{~s}, 3 \mathrm{H}, \mathrm{CO}_{2} \mathrm{Me}\right), 2.80-2.75(\mathrm{~m}, 1 \mathrm{H}), 2.59-2.51(\mathrm{~m}, 4 \mathrm{H})$, $2.48-2.43(\mathrm{~m}, 1 \mathrm{H}), 2.12(\mathrm{dd}, J=13.1,7.4 \mathrm{~Hz}, 1 \mathrm{H}), 1.58(\mathrm{~s}, 3 \mathrm{H}$, $\mathrm{Me}), 1.53$ (s, 3H, Me), 1.49 (s, 3H, Me), 1.07 (t, $J=7.4 \mathrm{~Hz}$, $3 \mathrm{H}) ;{ }^{13} \mathrm{C} \mathrm{NMR}\left(125 \mathrm{MHz}, \mathrm{CDCl}_{3}\right) \delta 205.6,200.3,171.5,169.9$, 139.1, 134.0, 84.1, 60.3, 60.1, 53.1, 50.5, 36.1, 33.5, 31.0, 30.3, 23.7, 22.5, 7.8; IR (neat): 2900, 1760, 1720, 1670, 1620, $1420 \mathrm{~cm}^{-1}$; HRMS $(\mathrm{m} / \mathrm{z})$ : $[\mathrm{M}]^{+}$calcd for $\mathrm{C}_{18} \mathrm{H}_{24} \mathrm{O}_{6}, 336.1573$; found, 336.1576 .

\section{Supporting Information}

\section{Supporting Information File 1}

Copies of ${ }^{1} \mathrm{H}$ and ${ }^{13} \mathrm{C}$ NMR spectra.

[https://www.beilstein-journals.org/bjoc/content/

supplementary/1860-5397-14-185-S1.pdf]

\section{Acknowledgments}

G. H. M. Rao thanks Prof. Faiz Ahmed Khan for his guidance, and the Department of Science and Technology (DST), New Delhi, for financial assistance.

\section{References}

1. Cardona, L.; Pedro, J. R.; Serrano, A.; Muñoz, M. C.; Solans, X. Phytochemistry 1993, 33, 1185-1187. doi:10.1016/0031-9422(93)85046-T

2. Décostered, L. A.; Hostettmann, K.; Stoeckli-Evans, H.; Msonthi, J. D. Helv. Chim. Acta 1987, 70, 1694-1702. doi:10.1002/hlca.19870700705

3. Jayasuriya, H.; McChesney, J. D.; Swanson, S. M.; Pezzuto, J. M. J. Nat. Prod. 1989, 52, 325-331. doi:10.1021/np50062a018

4. Morita, H.; Fujiwara, M.; Naotoshi, Y.; Kobayashi, J. Tetrahedron 2000, 56, 5801-5805. doi:10.1016/S0040-4020(00)00530-5

5. Miyaoka, H.; Tamura, M.; Yamada, Y. Tetrahedron 2000, 56, 8083-8094. doi:10.1016/S0040-4020(00)00730-4

6. Huang, J.-m.; Yokoyama, R.; Yang, C.-s.; Fukuyama, Y. Tetrahedron Lett. 2000, 41, 6111-6114. doi:10.1016/S0040-4039(00)01023-6

7. Mehta, G.; Singh, S. R. Angew. Chem., Int. Ed. 2006, 45, 953-955 doi:10.1002/anie.200503618

8. Inoue, M.; Sato, T.; Hirama, M. J. Am. Chem. Soc. 2003, 125, 10772-10773. doi:10.1021/ja036587+

9. Shi, L.; Meyer, K.; Greaney, M. F. Angew. Chem. 2010, 122, 9436-9439. doi:10.1002/ange.201005156

10. He, W.; Huang, J.; Sun, X.; Frontier, A. J. J. Am. Chem. Soc. 2008, 130, 300-308. doi:10.1021/ja0761986

11. Birman, V. B.; Danishefsky, S. J. J. Am. Chem. Soc. 2002, 124 , 2080-2081. doi:10.1021/ja012495d

12. Meng, Z.; Danishefsky, S. J. Angew. Chem. 2005, 117, 1535-1537. doi:10.1002/ange.200462509

13. Kouno, I.; Mori, K.; Kawano, N.; Sato, S. Tetrahedron Lett. 1989, 30 , 7451-7454. doi:10.1016/S0040-4039(00)70722-2

14. Kouno, I.; Mori, K.; Okamoto, S.; Sato, S. Chem. Pharm. Bull. 1990, 38, 3060-3063. doi:10.1248/cpb.38.3060

15. Wang, R.; Shimizu, Y. J. Chem. Soc., Chem. Commun. 1990, 413-414. doi:10.1039/c39900000413

16. Ghosh, S.; Sinha, S.; Drew, M. G. B. Org. Lett. 2006, 8, 3781-3784. doi:10.1021/ol061377y

17. Crimmins, M. T.; Jung, D. K.; Gray, J. L. J. Am. Chem. Soc. 1992, 114 , 5445-5447. doi:10.1021/ja00039a077

18. Corey, E. J.; Su, W. G. J. Am. Chem. Soc. 1987, 109, 7534-7536. doi:10.1021/ja00258a050

19. Corey, E. J.; Su, W.-g. Tetrahedron Lett. 1988, 29, 3423-3426. doi:10.1016/0040-4039(88)85179-7

20. Scarborough, R. M., Jr.; Toder, B. H.; Smith, A. B., III. J. Am. Chem. Soc. 1980, 102, 3904-3913. doi:10.1021/ja00531a037 
21. Smith, A. B., III; Boschelli, D. J. Org. Chem. 1983, 48, 1217-1226. doi:10.1021/jo00156a015

22. Hudlicky, T.; Govindan, S. V.; Frazier, J. O. J. Org. Chem. 1985, 50, 4166-4171. doi:10.1021/jo00221a043

23. Wolnisky, J.; Wolf, H.; Gibson, T. J. Org. Chem. 1963, 28, 274-275. doi:10.1021/jo01036a541

24. Niwa, H.; Wakamastu, K.; Hida, T.; Niiyama, K.; Kigoshi, H.; Yamada, M.; Nagase, H.; Suzuki, M.; Yamada, K. J. Am. Chem. Soc. 1984, 106, 4547-4552. doi:10.1021/ja00328a041

25. Ernst, A. B.; Fristad, W. E. Tetrahedron Lett. 1985, 26, 3761-3764. doi:10.1016/S0040-4039(00)89244-8

26. Curran, D. P.; Chang, C. T. J. Org. Chem. 1989, 54, 3140-3157. doi:10.1021/jo00274a034

27. Kraus, G. A.; Landgrebe, K. Tetrahedron 1985, 41, 4039-4046. doi:10.1016/S0040-4020(01)97182-0

28. Lange, J. H. M.; Klunder, A. J. H.; Zwanenburg, B. Tetrahedron Lett. 1989, 30, 127-130. doi:10.1016/S0040-4039(01)80342-7

29. Isawa, S.; Yamamoto, M.; Kohmoto, S.; Yamada, K. J. Org. Chem. 1991, 56, 2849-2853. doi:10.1021/jo00008a048

30. Hibbs, D. E.; Hursthouse, M. B.; Jones, I. G.; Jones, W.; Malik, K. M. A.; North, M. J. Org. Chem. 1999, 64, 5413-5421. doi:10.1021/jo990140v

31. Mandal, S. K.; Amin, S. R.; Crowe, W. E. J. Am. Chem. Soc. 2001, 123, 6457-6458. doi:10.1021/ja005568m

32. Khan, F. A.; Dash, J.; Sahu, N.; Sudheer, C. J. Org. Chem. 2002, 67, 3783-3787. doi:10.1021/jo025521e

33. Rao, G. H. M.; Khan, F. A. Synth. Commun. 2018, 48, 318-322. doi:10.1080/00397911.2017.1401638

34. Chatterjee, A. K.; Choi, T.-L.; Sanders, D. P.; Grubbs, R. H. J. Am. Chem. Soc. 2003, 125, 11360-11370. doi:10.1021/ja0214882

35. Chatterjee, A. K.; Morgan, J. P.; Scholl, M.; Grubbs, R. H. J. Am. Chem. Soc. 2000, 122, 3783-3784. doi:10.1021/ja9939744

36. Galan, B. R.; Kalbarczyk, K. P.; Szczepankiewicz, S.; Keister, J. B.; Diver, S. T. Org. Lett. 2007, 9, 1203-1206. doi:10.1021/ol0631399

37. Abbas, M.; Leitgeb, A.; Slugovc, C. Synlett 2013, 24, 1193-1196. doi:10.1055/s-0033-1338425

38. Epoxidation of enone 11 with $30 \%$ aq $\mathrm{H}_{2} \mathrm{O}_{2}$ solution using $\mathrm{NaHCO}_{3}$ afforded a 6:1 of two diastereomers (determined by ${ }^{1} \mathrm{H}$ NMR analysis of the crude reaction mixture) of $\alpha, \beta$-epoxy ketone in very low yield $(10 \%)$. Changing the base from $\mathrm{NaHCO}_{3}$ to aq $\mathrm{NaOH}$ solution $(1 \mathrm{~N})$ did not improve the yield. Then the diastereomeric mixture of $\alpha, \beta$-epoxy ketone was subjected to nucleophilic addition of Grignard reagent derived from 2-bromopropene in the presence of Cul led to the formation of an intractable mixture.

\section{License and Terms}

This is an Open Access article under the terms of the Creative Commons Attribution License (http://creativecommons.org/licenses/by/4.0). Please note that the reuse, redistribution and reproduction in particular requires that the authors and source are credited.

The license is subject to the Beilstein Journal of Organic Chemistry terms and conditions:

(https://www.beilstein-journals.org/bjoc)

The definitive version of this article is the electronic one which can be found at:

doi:10.3762/bjoc. 14.185 